\title{
ECONOMIC APPRAISAL OF ASTHMA AND COPD CARE: A LITERATURE REVIEW 1980-1991
}

\author{
Maureen P. M. H. Rutten-Van Mölken ${ }^{1}$, Eddy K. A. Van Doorslaer ${ }^{2}$ and \\ Frans F. H. RutTen ${ }^{2}$ \\ 'Department of Health Economics, University of Limburg, P.O. Box 616, 6200 MD, Maastricht, \\ The Netherlands and ${ }^{2}$ Institute for Medical Technology Assessment, Erasmus University, Rotterdam, \\ The Netherlands
}

\begin{abstract}
Despite the considerable burden and costs of illness and despite the increasing need to set priorities on the basis of efficiency considerations, only 20 economic appraisals of asthma and COPD care have been published during the past 11 years. This paper provides a detailed summary of the cost-effectiveness 'evidence' given by these studies and a discussion of relevant methodological issues. The studies comparing programme costs of delivery methods for oxygen and for aerosol bronchodilator drugs, provide the most straightforward evidence in favour of the concentrator and the metered dose inhaler respectively. There also seems to be evidence in favour of hospital-based home care programmes as compared to community-based home care programs. Health education, especially directed at asthmatic children seems to reduce health care costs and improve attitude, compliance behaviour and self-management skills. Information on the cost-effectiveness of pharmacothcrapy and diagnostic technologies, both important interventions in asthma and COPD, was found to be totally lacking.
\end{abstract}

Key words - COPD, asthma, economic evaluation, review

\section{INTRODUCTION}

The pressing need for more efficient allocation of resources in health care has stimulated interest in economic evaluation studies. Whereas the first studies tended to concentrate on the most visible applications of modern advanced diagnostic and therapeutic technology in medicine, the focus is gradually shifting to more routinely applied 'small-ticket' technologies. Although these are less expensive per unit of output, they often lead to much higher costs, because of their wide application in much larger patient populations, who often need long-term care. The treatment of asthma and chronic obstructive pulmonary disease (COPD) is characterized by such long-term, smallticket, mostly drug therapy.

The terminology of these two diagnoses is often confusing. In clinical practice patients frequently have overlapping characteristics and are not easily categorized as having either asthma or COPD. The Dutch practice is to treat asthma and COPD as different expressions of the same underlying disease called chronic non-specific lung disease (CNSLD). However we will follow the Englishlanguage literature and report separately on asthma and COPD. COPD refers primarily to chronic bronchitis and emphysema. It causes progressive loss of lung function and is primarily related to long term cigarette smoking, age, occupational exposure and airways hyper-responsiveness. Asthma is characterized by attacks of dyspnea or wheezing, reversible airflow obstruction and hyper-responsiveness [1].
In the Netherlands, the two conditions impair the quality of life of $10-20 \%$ of men and $5-10 \%$ of women and children. The associated burden of illness is considerable. The annual costs to society in the Netherlands are estimated to exceed 1 billion Dutch guilders. This includes health care costs and costs due to absence from work [2]. In the Netherlands, most asthma and COPD patients are treated by general practitioners (GPs). Almost $9 \%$ of all GP consultations and about $13 \%$ of all absenteeism due to illness is caused by asthma and COPD [2]. The ageing of the population will almost certainly lead to even larger costs of illness, especially due to COPD, in the near future. In children of 4-12 years of age asthma is the main reason for absence from school [3]. Also the number of hospitalizations due to asthma has been shown to be increasing significantly in children aged 1-4 [4]. Although they are generally considered to be of greater importance as a cause of disability and ill health than as a cause of death, asthma and COPD directly or indirectly cause $15 \%$ of all male mortality and $6 \%$ of all female mortality.

Since the prevalence and associated burden and costs of asthma and COPD are large and are expected to grow in the near future, it seems important to establish the cost-effectiveness of the various health care interventions which are in common use. Economic appraisals, by systematically comparing both the costs and the outcomes of alternative health care programmes, provide information on how to allocate scarce resources to obtain maximum positive health effects from those programmes. This article will review existing knowledge on the cost-effectiveness of 
asthma and COPD treatment and assess the quality of this information in a critical and constructive way. After describing the literature search, we review the topics of the studies and describe the nature of the economic appraisals. Then we deal with the alternative treatments and what is known about their costeffectiveness. The next sections review the measurement of outcomes and cost, followed by a thorough assessment of the quality of the effectiveness evidence on which the economic avaluations are based. The last section contains the conclusions and a discussion.

\section{SELECTION OF THE LITERATURE}

A medline search for publications in English found 19 articles offering economic appraisals of a particular asthma or COPD-related health care intervention [5-23]. A publication from the U.S. Office of Technology Assessment was added to this [24]. A combination of the following entries was used: respiratory tract disease, obstructive lung disease, asthma, respiratory therapy, respiratory drug therapy, economics, costs and cost-analysis, cost-benefit analysis and economic evaluation. The entry 'cost-benefit analysis' applied to all of the articles. The studies were all published between January 1980 and December 1991 .

\section{NATURE OF THE ECONOMIC APPRAISALS}

Like Williams [25], who reviewed the psychosocial literature on COPD, we found that the majority of the economic evaluation studies published in international journals have been peformed in the United States. Two studies were performed in the U.K., 1 in Australia, 1 in Germany and 1 in Sweden.

A wide variety of COPD interventions have been subjected to an economic appraisal (see Table 1). Health educational programmes for adults and for children have been appraised relatively often. These programmes are primarily directed at asthmatics. Economic appraisals of pulmonary rehabilitation-a multidisciplinary programme which among other things includes physical exercise and health education--and of domiciliary oxygen therapy and home care, generally include patients with rather severe chronic airflow limitation. Aerosol bronchodilator delivery methods have been the subject of several studies involving asthma, COPD and other respiratory diseases. We have not found a single economic appraisal of pharmacotherapy, which is the main treatment in asthma and COPD.

Before we discuss the studies themselves, it is necessary to consider what constitutes an economic evaluation. Drummond et al. [26] make a useful distinction between partial evaluation and full economic evaluation. Whereas partial evaluation studies are restricted to either costs or outcomes, or consider no alternatives to the intervention being studied, in full economic evaluations some combination of costs is compared with some combination of outcomes for two or more alternatives. Depending on the way in which the outcomes are assessed, a full economic evaluation is labelled a cost-minimization analysis, a cost-effectiveness analysis, a cost-utility analysis or a cost-benefit analysis [26, 27]. All the articles reviewed have been labelled according to Drummond's classification (see last column of Table 1).

In a cost-minimization analysis the central question is restricted to "how to obtain the same outcome at the lowest cost?' Equal effectiveness is explicitly assumed, backed up by evidence from good quality studies. For example, the studies by Lowson et al. [15] and Scheffler et al. [24] concerning alternative ways of delivering oxygen and aerosol bronchodilator drugs, explicitly assumed that the alternatives were equally effective in terms of lung function and bronchodilation respectively. So the analyses concentrate on the assessment of the costs only.

In contrast, the studies by McKeon et al. [16] and Motwani et al. [17], who also restricted their analyses to the costs only, were partial evaluation studies classified as cost-analyses. Motwani et al. in comparing home care with institutional care, and McKeon et al. in comparing the current practice of oxygen delivery with a rationalized programme, did not explicitly assume the equal effectiveness of these alternatives. Had they provided evidence for assuming equal effectiveness, their studies would have been labelled cost-minimization analyses.

If the outcome is measured in units such as life years gained, walking distance, prevented reduction in forced expiration volumes or improvement in score on a quality of life scale, then the economic evaluation is called a cost-effectiveness analysis. For example, the study by Schmidt et al. [18] on mechanical ventilation for various groups of patients estimated that the costs per life year gained ranged from $\$ 460$ for asthma patients to $\$ 2155$ for patients suffering from chronic bronchitis and emphysema.

In a cost-utility analysis, benefits are expressed in quality adjusted life years (QALYs). A quality of life correction factor called utility is used to adjust life years for their quality. This utility reflects the preference value for a particular health state. Since improving the quality of life might be seen as the ultimate goal of most asthma and COPD therapies, cost-utility analysis would be very appropriate to evaluate them. Although this is often recognised, only Toevs et al. [20] have attempted to measure this improvement in QALYs or 'well years' as they call therin. They evaluated a behavioural programme directed at increasing compliance with a pulmonary rehabilitation programme and concluded that the programme produced a 'well year' at a unit cost of $\$ 24,256$. Also Hay et al. [12] in their study of AAT replacement therapy applied several values for the quality of a restricted activity day, but these values were not actually measured. Since QALYs can be seen as a common unit for measuring the outcome of diverse interven- 


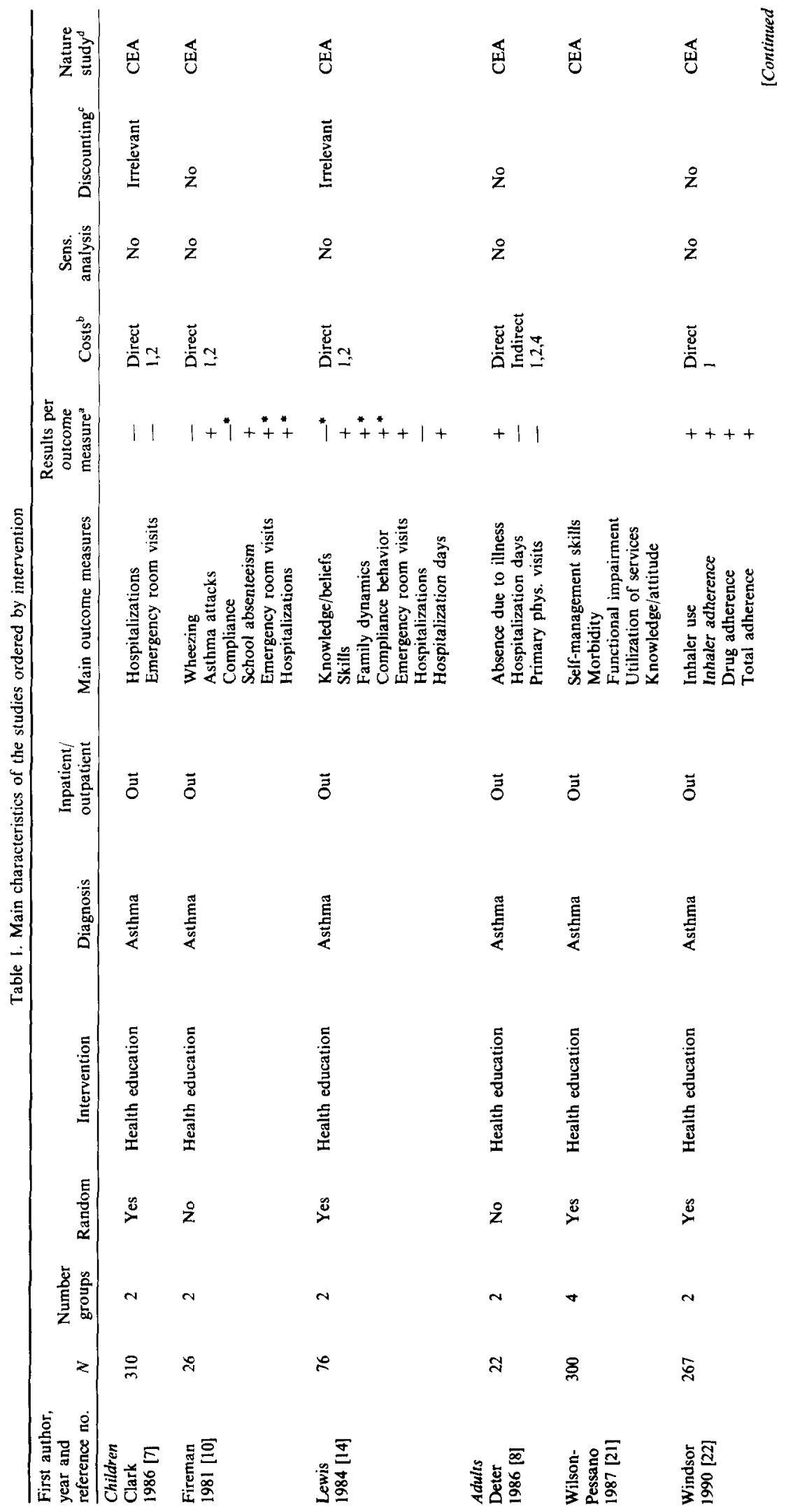




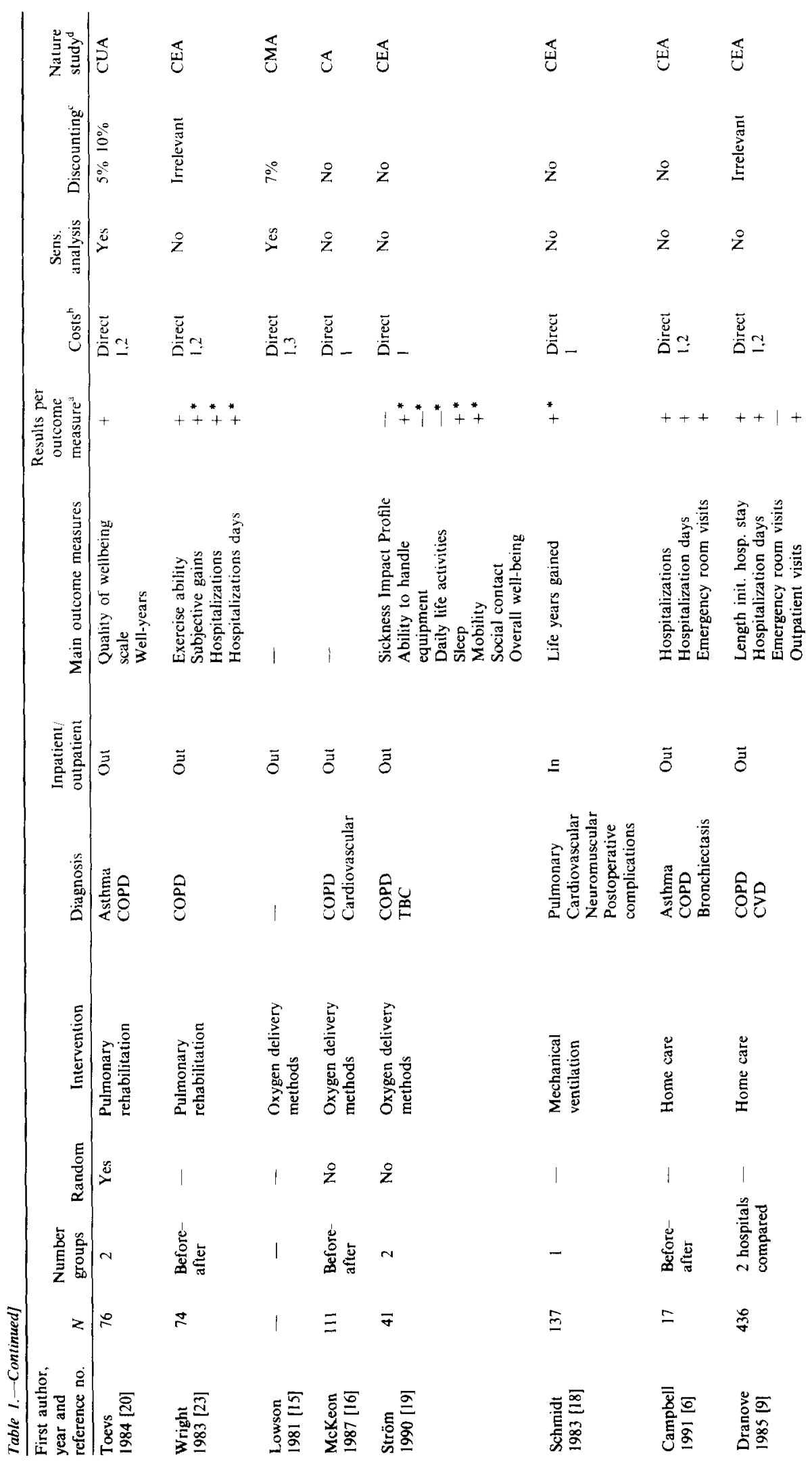




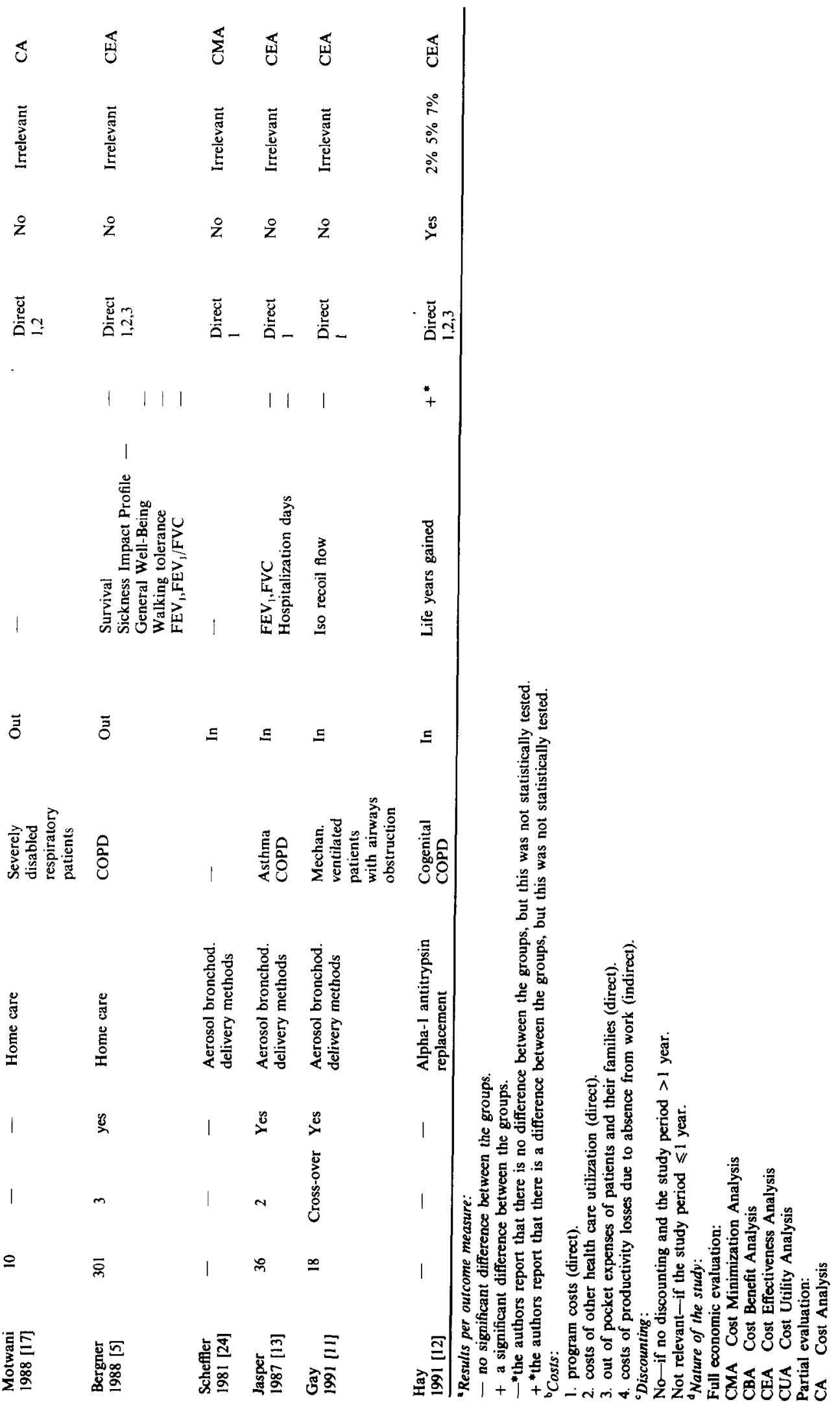


tions, cost-utility analysis has the potential additional advantage of allowing a comparison of interventions in asthma and COPD with those in other areas of the health care sector.

Finally, if the outcome is measured in monetary units, the evaluation is labelled a cost-benefit analysis. This is often limited to a comparison of items that can easily be expressed in money terms, such as the number of outpatient visits or the number of hospitalizations. Reviewing the literature, it became clear that there is a tendency to describe as cost-benefit analyses studies which concentrate on outcomes merely in terms of savings elsewhere in the health care sector. For example, in the study by Clark et al. [7] a sample of 310 low income urban children was randomly divided into a control group and an experimental group which received health education to improve asthma management at home. The costs of delivering the health education programme were compared to the outcomes in terms of health care cost savings mainly consisting of a reduced number of hospitalizations and emergency room visits. Although expecting a positive influence, the effect of health education on the ultimate goal of the intervention, i.e. improvement in quality of life, was not measured. As Guyatt et al. [27] and Drummond et al. [28] have shown, a cost-benefit analysis involves taking into account more than just the outcomes easily expressed in monetary units. It involves the monetary measurement of all relevant final outcomes, including health improvements. In fact none of the studies reviewed could be described as a full social cost-benefit analysis.

In addition to Drummond's classification, economic appraisals can be divided into empirical economic evaluation studies and studies based on modelling. In empirical evaluation studies, the economic analysis is linked to a clinical study. Modelling studies are in fact secondary analyses in which cost and outcome data obtained from several previous studies are combined into an overall cost-effectiveness model. The studies by Lowson et al. and Hay ct al. are good examples of the latter.

\section{ALTERNATIVES COMPARED}

Since economics is about choice, the point of departure for an economic evaluation is to specify the alternatives. In all studies, a comparison is made between two or more alternatives, although they are not always explicitly given. In 7 studies the alternative was 'doing nothing', which can be described more precisely as 'continuing conventional or usual care'.

\section{Health education for adults}

Health educational programmes are often compared with 'doing nothing'. Here 'doing nothing' is considered to be a reasonable alternative since patients participate in such programmes alongside their usual, mainly pharmacotherapeutic treatment without fear of an acute situation if the programme is omitted. Since adherence to long term pharmacotherapy is known to be low in asthma and COPD patients, Windsor et al. [22], developed a standardized health education programme to increase therapy adherence. At 12 month follow-up, the experimental group of patients $(n=132)$ showed a significantly higher level of improvement in correct inhaler use, inhaler adherence, medication adherence (self reported and checked with a theophylline level test) and total adherence compared to the do-nothing control group patients $(n=135)$. Excluding intervention development costs, the programme costs $\$ 32.03$ per patient.

Deter [8] also compared the cost-effectiveness of health education combined with group coping therapy for working adult asthmatics with a do-nothing control group. He found a significant reduction in the number of working days lost and a non-significant reduction in the number of working days hospitalized and number of visits to the general practitioner, resulting in estimated savings of DM 1951 per patient.

Few educational efforts directed at adults seem to have been evaluated economically. In an attempt to change this situation Wilson-Pessano et al. [21] report their plans for a cost-effectiveness study in which 300 patients are to be randomly divided into 4 treatment groups: an individual self-management group, a group self-management group, a self-management information/attention control group and a data only control group. The emphasis will be on self-management practices, morbidity, functional impairment, medical regimen and the utilization and costs of services.

\section{Health education for children}

Educational programmes in children have been studied more widely. Three of these studies also considered cost-effectiveness [7, 10, 14]. All three, by comparing programme costs with savings due to a decrease in emergency room visits, hospitalizations and drug use, reached the conclusion that educational programmes for children are cost-effective. When compared to a 'do-nothing' control group Fireman et al. [10] have shown that health education directed at both the child and the parents resulted in a significant reduction in asthma attacks, school absenteeism, emergency room visits and hospitalizations. Compliance with drug therapy was also better. The costs of additional drug use and the costs of the educational programme provided by a nurse-educator were half the savings due to reduced hospitalizations and emergency room visits, savings of $\$ 2920$ and $\$ 225$ per child per year respectively. In a randomised trial, Lewis et al. [14] compared a detailed, small-group asthma care training programme directed at both children and their parents $(n=48)$ to 
a control therapy consisting of a few large group sessions ( $n=28$ ). They estimated savings of approximately $\$ 180$ per child per year for those in the experimental group. Besides a significant reduction in emergency room visits and hospitalizations, they also found a significant reduction in parents' reports of children's dependency on adults for care and of children's troublc with asthma as well as improved family communication. In the study by Clark et al. [7], no significant reductions in hospitalizations and emergency room visits were found when the complete experimental and 'do-nothing' control groups were compared. However when the comparison was restricted to two subgroups, one of children with at least one hospitalization in the year preceding the intervention, the other to children with at least 1 hospitalization and 6 emergency room visits in the previous year, significant reductions and savings were found. The authors might have drawn the conclusion that health education may only be costeffective when directed at severely afflicted patients with at least one hospitalization a year. However they did not do so, because positive effects in terms of improved self management skills, school performance and school adjustment reported elsewhere have shown health education to be effective for wider groups.

\section{Pulmonary rehabilitation}

The continuation of the situation before the programme is the alternative to which Wright et al. [23] compared their pulmonary rehabilitation programme. Pulmonary rehabilitation can be defined as an individually tailored, multidisciplinary programme which, through accurate diagnosis, therapy, emotional support and education, stabilizes or reverses the progress of the disease and seeks to return the patient to the highest possible functional capacity [29]. Wright et al. estimated the savings achieved by pulmonary rehabilitation due to the reduction in hospital days in the first year following the programme to be $\$ 217,610$ for a group of 57 patients for whom follow-up data were available. Exercise ability also increased. The costs of the programme amounted to $\$ 66,462$, resulting in net savings of $\$ 151,148$ per year for these 57 patients.

Providing exercise programmes and prescribing daily exercise alone is not enough. As with drugs, non-compliance with exercise is a common problem. Toevs et al. [20] studied the effects of using behavioural programmes to improve compliance with an exercise programme. The control group was assigned an exercise programme but did not receive a behavioural programme. Adding the behavioural programme to the exercise programme produced one additional quality adjusted life year at a cost of $\$ 24,256$ per patient. Assuming that the effects will last for 2.5 years, the cost per QALY drops to $\$ 18,455$.

\section{Domiciliary oxygen therapy}

'Doing nothing' is not a realistic alternative for oxygen therapy. Repeated hospitalizations or in the long run even admission to a nursing home could become necessary in the absence of this therapy. A study comparing oxygen therapy with the above mentioned alternatives is unlikely to be designed and conducted because of the overwhelming acceptance of oxygen therapy [30]. Its efficacy in terms of reduced mortality and improved neuropsychological function has been established by several clinical trials. Since it does not seem necessary to ask whether domiciliary oxygen is worthwhile per se, studies of oxygen therapy are concerned with the alternative ways of delivering the oxygen. In three studies $[15,16,19]$ concentrator oxygen-a concentrator is an electrically driven machine which extracts oxygen from the air-has been compared to gas cylinder oxygen in order to determine the most efficient way of delivering oxygen at home. The first of these studies also added liquid oxygen to the comparison. Lowson et al. [15] explicitly state that the alternatives under consideration seem to be equally effective. This is confirmed by the finding of Ström et al. [19] that scores on the Sickness Impact Profile did not significantly differ between the groups treated with gas cylinder oxygen and concentrator oxygen. Although quite different in their methodology and calculation of costs, all three studies reached the overall conclusion that in most situations the concentrator was the least expensive method of delivering oxygen. In addition Ström et al. found that concentrators were easier and safer to handle and can be managed by the patient him or herself in most cases.

Unlike cylinders, concentrators cannot always be hired, and the oxygen delivery and maintenance cannot thus be provided by the manufacturer. In that case large capital outlays may be needed from the health care sector at the start of a concentrator oxygen project. This is not only due to the high purchase price of the equipment but also to the need to establish a workshop facility from which the concentrators are maintained [15]. Where these large capital costs apply, concentrator oxygen therapy is more cost-effective the larger the number of patients served by this unit. Renting or purchasing concentrators seems to make no difference to maintenance costs [16].

\section{Home care}

Home care is usually compared to alternatives which may well be substituted for it, such as institutional care or doctor's office care $[5,6,9,17]$. Two studies [5,9] found no significant reductions in health care costs due to home care. Bergner et al. [5] randomly assigned homebound COPD patients aged 40-75 to one of three groups: respiratory home care $(n=99)$, standard home programme $(n=102)$ or doctor's office care without additional home nursing 
services $(n=100)$. They found no significant difference between the three groups in survival, pulmonary function, walking ratc, activitics of daily living, and scores on the General Well Being and the Sickness Impact Profile. The home care programmes even lead to additional health service utilization rather than to the substitution of ambulatory care for inpatient care. Also, the imputed costs for unpaid help for the respiratory and standard home care groups did not differ significantly from those for the office care group. The reason no cost reduction was found may be that Bergner et al. studied community-based home care programmes. Providing these programmes, as compared to hospital-based home care programmes, may imply less communication between community based nurses and the treating physician about services that could be substitutes for regular check ups at the hospital.

Dranove [9], by comparing two hospitals, one with and one without a home care programme, showed that home care significantly substitutes for the last few days of a hospital stay and outpatient visits. Home care reduced the initial length of stay of patients older than 65 years suffering from cerebrovascular disease (CVD) or COPD by 3.4 days and the number of clinic visits by 6.2 per patient. No reduction in the number of emergency room visits was found. Despite this substitution Dranove found no significant reduction in overall hospital costs because of the relatively larger costs of the home care programme.

Probably home care is more cost-effective in highly selected patient populations. For example, Campbell et al. [6] studied the effects of a hospital hased home care programme in severe asthma, COPD or bronchiectasis patients showing substantial co-morbidity and frequent hospitalizations due to pulmonary disease. An additional inclusion criterion was the presence of a significant other in the home or community willing to assume the role of caretaker. When pre-programme costs were compared to programme costs, the overall health care savings were $\$ 328$ per patient per month. Similarly, for respirator-dependent patients needing around-the-clock home care, Motwani et al. [17] found the costs of home care to be lower than the cost of care in the respiratory centre which was considered the only possible alternative for these patients.

\section{Aerosol delivery of bronchodilator drugs}

Two studies reported on the cost-effectiveness of an updraft nebulizer compared to a metered dose inhaler as alternative methods to delivery aerosol bronchodilator drugs in hospitalized patients [11, 13]. Both studies included the same indirect costs e.g. labour costs, medication costs and the cost of the devices. The randomized study by Jasper et al. [13] involved patients with exacerbated obstructive lung disease, most of whom were transferred to the pul- monary ward from the emergency room or intensive care units. They received bronchodilation treatment with metaprotenerol via therapist administered updraft nebulization or via metered dose inhaler. The randomized cross-over study by Gay et al. [11] involved intubated mechanically ventilated patients, some of whom may not have had obstructive lung disease [10]. In the case of the intubated patients the nebulizer and the metered dose inhaler were attached to endotracheal tubes. Whereas Gay et al. performed a single treatment comparison, Jasper et al. assessed not only immediate response to bronchodilator drugs but also response during hospitalization and response at discharge from the hospital. In the first study no differences were found between the delivery methods so far as iso-recoil flows or side cffcets in terms of an increased heart rates. Jasaper et al. found no differences between the delivery methods with respect to initial post-bronchodilator and discharge post-bronchodilator $\mathrm{FEV}_{1}$ (Forced Expiratory Volume in one second) and FVC (Forced Vital Capacity) and duration of hospital stay. In addition, both studies found that the metered dose inhaler was more cost-effective than the updraft nebuliser, and may be substituted for it. According to Jasper et al. such a substitution would not be appropriate for patients in the intensive care unit or the emergency room. However, the 1990 study by Gay et al. showed that the metered dose inhaler was also morc cost-cffective for these patients.

The 1981 Office of Technology Assessment report by Scheffler et al. [24] contained estimates of the cost of delivering aerosol medication in hospitalized patients using three different devices: an intermittent positive pressure breathing machine (IPPB), an ultrasonic nebulizer and a simple aerosol generator. Equal effectiveness was assumed and supported by referring to several studies. The direct costs of labour, equipment, disposables and electricity were considered. Again simple aerosol treatment proved to be the least costly both as initial treatment and follow-up treatment.

\section{Mechanical ventilation}

When the IPPB is used for mechanical ventilation purposes where a patient's breathing has totally ceased owing to the suppresion of brain activity, paralysis of the respiratory muscles, chest wall injury or acute and potentially reversible lung disease, its effectiveness is well accepted, and is similar to that of other mechanical aids to support the ventilatory function. In such cases 'doing nothing' would inevitably lead to immediate death. Although the method is well-accepted, it remains a problem to select the patients most likely to benefit from prolonged mechanical ventilation. Schmidt et al. [18] showed in their study that the cost of extending life for one year with mechanical ventilation varied from $\$ 2155$ for COPD to $\$ 8026$ per year of extended life for cardiac patients. Cost-effectiveness ratios were 
considerably lower for patients with asthma and COPD largely because the mortality during hospitalization was lower. Only the total non-physician charges of hospital care were included and a portion of the costs of the non-survivors in a diagnostic group was added to the costs of survivors in that group.

\section{Alpha-1 Antitrypsin Replacement Therapy}

Congenital Alpha-1 Antitrypsin (AAT) deficiency results in COPD. AAT can be replaced intravenously and this replacement therapy - the costs of which are estimated to be $\$ 30,000$ per year-has been shown to improve levels of AAT in serum and epithelial tissue. However, clinical efficacy in slowing down COPD progression is unknown. Conducting a controlled trial would take a very long time due to the small number of potential beneficiaries. Therefore Hay et al. [12] took available data on congenital COPD incidence, prevalence, survival and costs of COPD treatment and applied it to a cost-effectiveness model of AAT replacement therapy in homozygous AATdeficient COPD patients. A range of hypothetical efficacy values (expressed as a percentage of the survival rate of the normal population) was compared to the cost of AAT replacement therapy and other lifetime health care costs after the diagnosis of COPD. At an efficacy of $70 \%$, the costs per life year saved would be between $\$ 28,000$ and $\$ 72,000$ depending on patient age, sex and smoking status. Quality of life adjustment does not alter these results drastically.

\section{OUTCOMES}

Sixteen of the 20 studies examined both the cost and the outcomes, using a great variety of outcome measures which can be classified into four main categories. More detailed information on the outcome parameters used and the significance of the effects measured by them can be found in the column 'main outcome measures' and 'results per outcome measure' of Table 1.

\section{Clinical measures}

Clinical measures include physiological measures such as lung function parameters, physical measures such as exercise tolerance and walking distance, and laboratory assessments. Lung function parameters are essential for monitoring the course of asthma and COPD, because of their known relationship to disease prognosis [31]. However, interventions such as health education, pulmonary rehabilitation and home care $[7,8,32]$ do not seem to alter spirometric data. Therefore, most of the studies dealing with these interventions do not include lung function parameters. Relevant effects of health education are usually measured in terms of increased knowledge, improved skills, improved attitude towards asthma and reduced health care utilization. The effects of pulmonary rehabilitation are usually measured in terms of improved oxygen consumption and physical measures such as walking distance and exercise tolerance [23].

An evaluation confined to a fairly narrow assessment of physiological status, as in Jasper et al. [13] gives little information about patients' overall functioning. Moreover, measures such as walking distance look meaningful and easy to analyze, but may bear only a loose relation to the patient's underlying condition. It has been shown that a therapy which produces changes in clinical status may not produce corresponding changes in quality of life [33-40]. Other factors such as the patient's attitude, expectations, emotions, ability to adapt, and sociological factors may intervene. Since it is likely that clinical and quality of life measures address different aspects of the disease, a meaningful outcome assessment should include both.

\section{Disease-specific quality of life measures}

The studies which did use quality of life measures of outcome such as Lewis et al. [14] and Ström et al. [19], who even developed a study-specific questionnaire, generally present a pattern of different aspects of quality of life directly related to having the particular disease. Such a heterogeneous description of changes in disease-specific quality of life may be sufficient, particularly when the results all point in the same direction and no comparison with interventions in other patient populations is envisaged. The rationale for using such disease-specific outcome measures lies in their potential for increased responsiveness. Although disease-specific measures may be more sensitive to disease-specific changes, they lack general reference points which enable across-programme comparisons.

\section{Generic quality of life measures}

In order to allow such comparisons and in order to facilitate the interpretation of the heterogeneous results one may want to combine them into a few subdimension scores or into one overall summary score, called a health profile and a health index respectively. Both Ström et al. [19] and Bergner et al. [5] attempted to quantitatively assess the relative importance of their information using a measure of the overall quality of life such as the SIP. The SIP is a generic measure of health-related dysfunction in 12 areas of activity: ambulation, mobility, body care and movement, social interaction, emotional behaviour, alertness behaviour, communication, work, eating, sleep and rest, household management and recreation and pastimes. Besides 12 category scores, it provides an overall score, a psychosocial dimension score and a physical dimension score. 


\section{Utilities and $Q A L Y S$}

The primary objective of indices and profiles is an overall summary description of health status. One may go one step beyond the description by explicitly valuing the summarized descriptions of the changes in health status. This is particularly necessary when measures of different dimensions of the quality of life are of different size or sign. By means of utility measurement such positive and negative effects may be evaluated, balanced and integrated in an overall value for the quality of life, the so called utility. Toevs et al. [20], who used the Quality of Well-Being Scale developed by Kaplan and Bush [41, 42], converted the quality of life gains into a single index of so-called 'well-years' or QALYs. At baseline and follow-up each patient's health state was classified into 1 of 43 mutually exclusive levels of functioning. The levels were obtained by combining different levels of mobility, physical activity, social activity and different symptomatic disturbances. Then utilities were assigned to each patient's health state. These utilities, which reflect the preference weights for the various health states, were elicited from the general population. Finally each patient's utility was multiplied by the number of years the effects were expected to last. This technique is relatively new and still rarely used in the domain of chronic diseases, because it is expected to be insensitive to slight improvements (or reductions) in health status [43]. But, particularly because economic evaluation studies aim at providing information for overall comparisons between interventions, QALYs are promising outcome measures, even though a number of methodological problems need to be solved. However, the study by Toevs et al. shows this approach to be feasible in evaluating treatment for a common chronic condition such as COPD.

Sometimes the outcome assessment is restricted solely to the intermediate effects of treatment on reductions in health services use. Although these outcomes can be measured solely in volume terms, they are usually measured in monetary units and incorporated in the numerator of a cost-effectiveness ratio, by the calculation of net incremental costs. The same is true for absence from work due to illness, although the costs associated with sick leave are often presented separately. Therefore we describe them more thoroughly in the next section. Like absence from work in adults, school absenteeism is an important outcome measure in studies involving children. Only Fireman et al. [9] studied the impact of health education on school absentccism, finding that it was reduced ten-fold.

\section{$\operatorname{cosTS}$}

In the reviewed studies four main categories of costs could be identified:
Costs of organizing and operating a particular health care programme

The studies dealing with alternative ways of delivering aerosol bronchodilation [11, 13, 24] all adopt an administrator's perspective in which only programme costs are considered. All found that delivering medication through a metered dose inhaler is the least expensive, mainly because of lower labour costs but also because of lower equipment costs. The comparison of alternative ways of delivering oxygen is also restricted to programme costs $[15,16,19]$. Ström et al. [19] even restricted their analysis to annual running cost per patient-which were twice as high for gas cylinders as for concentrators-without considering set-up costs. However set-up costs can be very important, as can be seen from the studies of domiciliary oxygen therapy, by McKeon et al. [16] and Lowson et al. [15], which were described above.

In evaluating alternative ways of delivering aerosol bronchodilation or oxygen, a restriction to programme costs may be justified, since equal effectiveness between the delivery methods is assumed and no change in the use of other health services is to be expected. However in the study by Windsor et al. [22] of health education this may be too limited a scope. If health education is as effective in increasing medication compliance as Windsor et al. showed, than a decrease in the use of other health care services such as visits to doctors' surgeries may be expected and should have been measured.

When calculating costs associated with providing a particular programme, resources that are used solely for the purpose of the evaluation study itself have to be identified and subtracted from the program costs, but few studies do so. Clark et al. [7] and Lewis et al. [14] are exceptions, in separating the costs of evaluating the educational programmes from routinely incurred costs. Unfortunately, these studies also excluded programme devclopment costs, which should have been included.

\section{Costs of utilization of other health services}

With the exception of the study by Windsor et al., all the studies concerning health education $[7,8,10,14,21]$ or home care $[5,6,9,17]$ do include the costs of other health services. They are particularly interested in savings as a result of reduced emergency room visits, clinic visits, and hospitalization days. The latter was of prime importance in the two studies dealing with pulmonary rehabilitation $[20,23]$.

It frequently occurs that the mean cost of hospitalization is very much affected by a small number of expensive cases. As a result, differences in the net costs of two programmes may be influenced by only a few cases. Since this reduces statistical power, testing differences in hospitalization costs is difficult.

Savings in health care resource use may be either compared with the programme costs or deducted 
from the programme costs in order to calculate the net cost of the intervention. Clarke et al. [7] used the first approach and found that for children who had been hospitalized during the year preceding the programme, health education reduced emergency room visits and hospitalizations, saving $\$ 11.22$ for every $\$ 1.00$ spent on health education. Toevs et al. [20] used the second approach and calculated the net costs of the intervention by deducting the hospital costs which were saved from the direct costs of the behavioural intervention programme.

\section{Out-of-pocket expenses of patients and their families}

The most comprehensive calculation of COPD health care costs is provided by Bergner et al. [5]. They include, from a societal viewpoint, all relevant inpatient and outpatient costs, which they obtained from several sources such as patients, bills, receipts, third party payers, pharmacists etc. Like Deter [8], they also included out-of-pocket payments by patients, mainly travelling expenses. A third study also included costs falling on patients, but a separate identification of these costs is not provided [15].

The loss of productivity due to absence from work,
imputed volunteer time or family time

The first three categories of costs are often referred to as direct costs. In economic evaluation jargon the term indirect costs refers primarily to production losses due to absence from work because of illness. Measuring the reduction in days of absence due to illness is especially relevant in asthmatic patients and patients with mild airflow limitation. Measuring the impact of treatment on employment status may not be very relevant for patients with severe chronic airflow limitation, since this mostly concerns relatively older patients who have only a few work years remaining and who would probably continue to decrease in work ability during those years because of their lung disease. Asthma and COPD interventions can only make an impact in terms of returning people to the work force for patients whose lung disease is identified at a younger age, when the disease is less progressive. Although relevant for less severely afflicted patients, measuring the impact of treatment on employment status may be difficult. Factors such as the national unemployment rate, duration of unemployment, age etc. may influence the percentage of patients returning to work. The study by Deter [8], which involved only working adult asthmatics, is the only one to consider savings due to a reduced number of working days lost. Loss of productivity due to absence from work on days of group therapy was included in programme costs. Production loss was estimated by means of individual real market wages. Earnings are commonly used but also frequently criticized measures of production losses, since it is questionable whether production is actually lost, especially in times of unemployment. Also, as a consequence of this approach, higher priority in the access to care would be given to high income earners. Perhaps a more realistic but difficult to perform alternative would be to consider the real costs to employers arising from the replacement and re-education and training of employees. Drummond et al. [26] advise that productivity gains and losses should not be included in calculating the net costs of an intervention because of the problem of the differential valuation of time for those in and those out of the labour force. It certainly seems preferable to count changes in productivity separately from other costs and outcomes. One may also measure restricted activity days for patients who are not in the work force and estimate their productivity loss by, for example, using the wages paid employees would get when performing the same work. Such a proxy is used by Bergner et al. [5], when they estimated the imputed costs of unpaid help by family or friends using the costs of home health aides.

\section{Costs vs expenditures and charges}

In general, costs refer to the sacrifice made when a given resource is consumed, and not to expenditures only. Wright et al. [23] should not have excluded the services for which members of the rehabilitation staff donated their time from the programme costs. Although donated time requires no expenditures, it does involve resource use, because these staff members cannot put their time into alternative services.

Although detailed costing methodology is often lacking it can be seen that a majority of studies still use average costs (per hospital day, per home nursing visit, average wages etc.) or charges. This may not reflect the real costs borne by society for a particular treatment. The average daily hospital costs are likely to be a poor guide for the savings in resource use if hospital stays were shortened, since resource use is typically higher in the first part of a patient's hospital stay [44]. Because home nursing can be a substitute only for the last few days of a hospital stay, Dranove et al. [9], in studying the impact of a home nursing department on the savings in hospital costs, thought it appropriate to include only the costs of routine care and clinical laboratory work in the average daily hospital cost. In comparing respiratory centre care with home care, Motwani et al. [17] showed that the actual costs of ventilator dependent respiratory centre care were much higher than Medicaid reimbursement rates. This was because higher levels of staff were required in the respiratory centre than in the other hospital departments, although the reimbursement rates were the same. Based on charges Motwani et al. concluded that home care was more cost-effective than respiratory centre care. When based on actual costs, home care would become even most cost-effective. Sometimes, using the amounts charged as a proxy for costs may not introduce a stystematic bias into identifying differences between 
treatment programmes, although it still risks misstating the magnitude of any cost differences.

An incremental analysis, as for example in Lowson et al. [15], does not consider costs which are common to both alternatives. It deals therefore with differential costs, rather than with the total treatment cost. An incremental analysis may be sufficient to support decisions concerning only the programmes studied. But if a comparison with other alternatives, perhaps outside the medical domain of the intervention is wanted, then total costs are needed.

\section{Discounting and sensitivity analysis}

Sound economic methodology also prescribes that costs and benefits occurring at different times should not just be added or subtracted, but rather expressed in terms of their equivalent values at one point in time. This adjustment procedure is called discounting and usually involves calculating the net present values of future costs and outcomes using the same discount rate. The discounting of health effects is not uncontroversial (for a recent debate see Refs [45-47]). In most of the studies, discounting was irrelevant, because no long term consequences were studied. Hay et al. [12], Lowson et al. [15] and Toevs et al. [20] have applied discounting. They also studied the influence of the application of various discount rates $(0 \%, 5 \%$, $10 \%$ ) in a sensitivity analysis. Both Lowson et al. and Toevs et al. concluded that the various discount rates have little influence on the cost-effectiveness ratios found in their studies because only a limited time period was considered. Discounting did have a larger effect on the evaluation of Alpha-1 Antitrypsin replacement therapy, because here lifetime treatment costs and outcomes were estimated [12]. In general, programmes that require current investments to achieve benefits far in the future are more strongly affected by the choice of a discount rate. Therefore a screening programme for Alpha-1 Antitrypsin deficiency could be even more affected by the choice of discount ratc than is Alpha-1 Antitrypsin replacement therapy itself.

Each study should perform a sensitivity analysis to show the influence of several assumptions on the overall cost-effectiveness ratio. Hay et al. [12] have provided an exemplary sensitivity analysis, in which they show the effects of a $40 \%$ increase and decrease of not only discount rates but also of annual costs of COPD care, the quality of a restricted activity day, the median survival from onset of COPD and annual costs of AAT treatment. The cost-effectiveness ratio proved to be most sensitive to the assumptions about the latter two factors.

As Guyatt et al. [27] point out, it is important to analyze the costs (and outcomes) from a broad perspective. This means the inclusion of all four categories of costs, as far as they are relevant. This may also mean the consideration of various viewpoints (government, Ministry of Health, patients, health care providers) if one wants to understand more fully the gains and losses to key actors in the system.

\section{QUALITY OF THE EFFECTIVENESS EVIDENCE}

It is argued by Drummond [48] that economic appraisals should be based on reliable evidence of effectiveness. It is certainly possible to perform a good economic appraisal without actually comparing two or more patient groups. But especially in that case, effectiveness information based on previous good quality studies should be provided. Good examples of such studies are the ones by Lowson et al. [15], Schmidt et al. [18] and Scheffler et al. [24]. If no such information is available a range of possible effectiveness levels should be considered to give some information on the sensitivity of the cost-effectiveness to these levels. This was done by Hay et al. [12]. However, Motwani et al. [17] used the opinion of 10 patients and their medical professionals: this would seem to be insufficient evidence to support the assumption that respiratory centre care was the only appropriate substitute for home care.

Ten out of the 20 economic appraisals actually compared two or more patient groups $[5,7,8,10,13,14,19-22]$, and in 7 of these 10 studies patients were randomly divided into an experimental and a control group [5, 7, 13,14, 20-22]. In experimental studies that concentrate on the impact of health education or pulmonary rehabilitation, an observed effect could partly be due to the attention the experimental group received. Giving a placebo to a control group in studies other than those concerning pharmacotherapy is considered to be difficult. However, Wilson et al. [21] have tried to control for the personal attention given to the experimental groups by designing a study which incorporates two control groups, one in which patients get the same written information as the experimental group but no personal attention, and one in which patients get no information or attention at all. The former is called an attention-placebo [27]. Lewis et al. also provided their control group with the same knowledge as the experimental group. However the knowledge was provided in impersonal large group sessions, whereas the experimental group received more personal attention in small groups.

Three studies were before-and-after comparisons of just one patient group $[6,16,23]$. Because of this design, the favourable outcomes which both Campbell et al. [6] and Wright et al. [23] found cannot solely be attributed to the intervention. Other factors such as the natural course of the disease or regression towards the mean might have explained at least part of the improvement.

The study by Gay et al. [11] used a cross-over design, which is used mainly to enhance the power of the statistical comparisons. In the case of different aerosol delivery methods, no carry-over effect (in 
which the effect of administering albuterol by means of a metered dose inhaler in the first period influences the effect of administering it by means of an updraft nebulizer in the second period) is likely, since a wash-out period of $4 \mathrm{hr}$ was allowed between the first and the second delivery.

Drawing conclusions based on a comparison of two health care institutions is extremely difficult because of the large number of biases that can occur. Dranove [9] tried to avoid the bias that might have resulted from the comparison of two hospitals-one with and the other without a home care programme - by taking younger patients who were not eligible for home care as a kind of control group. Instead of comparing the mean length of hospitalization for elderly patients in the two hospitals, he compared the difference in the length of hospitalization of younger and older patients in one hospital with the difference in the other hospital. Thus he partly controlled for differences between patient populations. Despite this, he could not completely rule out the possibility that differences between the hospitals affected his study results.

Some studies are of poor quality due to the inadequate sample size. These studies may not have the power to detect clinically significant differences. The fact that Deter [8] and Gay et al. [11] found no significant difference between the experimental and control group in terms of hospitalization days and visits to the primary physician may be due to the small sample size. Deter, moreover, divided patients $(n=57)$ randomly into an experimental and a control group before applying the inclusion criterion that patients had to be employed. Therefore only 22 patients remained, of whom only 7 patients in the experimental group and 8 in the control group finished the whole study. Large numbers of withdrawals can cause serious analysis problems, especially when withdrawal is due to a deterioration only occurring in the control group. When deterioration also leads to higher health care cost, the cost-effectiveness ratio will be overestimated. In the study by Clark et al. [7] more than $20 \%$ of the patients were lost to follow-up, and in the study by Bergner et al. [5] $21 \%$ were lost. Since neither provided a comparison of these patients with the patients who completed the study it is impossible to say whether this influenced the cost-effectiveness ratio in any way.

Obviously, the most reliable effectiveness evidence is that produced by a well-designed randomized controlled trial. Performing an economic evaluation alongside such a trial entails a number of problems. Even those trials which are directly concerned with assessing the effectiveness rather than the efficacy of the therapy may still be conducted under such rarefied experimental conditions that costs and outcomes will be quite different to those under normal conditions [49]. There remains a need to assess ther- apies as they are routinely delivered in actual daily, practice.

\section{CONCLUSIONS AND DISCUSSION}

Although central governments and third party payers increasingly stress the need to set priorities on the basis of efficiency considerations, there have been only 20 economic appraisals of asthma or COPD-interventions published in the last 11 years, most of them American. Because of the small number of studies per subject, the considerable differences in the measurement of both costs and outcomes and other methodological issues, only very preliminary conclusions can be drawn.

Health educational programmes seem to be relatively inexpensive and in children they seem to result in savings due to less hospitalizations and emergency room visits. Moreover there seem to be indications for improvements in attitude, self-management skills and school absenteeism. The study by Windsor et al. is the only economic appraisal dealing with health education in adults offering guidance for a cost-effective improvement in compliance behaviour. Toevs et al. also found evidence of increased compliance when a behavioural programme was added to an exercise programme. The costs per QALY for this intervention were more than $\$ 24,000$. The conclusions that can be drawn from the studies of inpatient aerosol drug delivery methods and oxygen delivery methods are perhaps the most straight-forward, the first indicating that the simple metered dose inhaler is the most cost-effective and the latter supporting the concentrator option, provided that the number of patients to be served is not too small. The results of the studies dealing with home care point in different directions. However, there seems to be evidence in favour of hospital-based home care programmes directed at highly selected, very severely afflicted patient groups, as compared to communitybased home care programmes in wide patient populations.

Physicians are responsible not only for providing an optimal therapy from a medical viewpoint, but also for providing an efficient therapy. More costeffectiveness evidence is required on the various asthma and COPD interventions described above. This is especially true for the relatively expensive treatments such as pulmonary rehabilitation and home care. Moreover, in two particular areas costeffectiveness evidence seems to be totally lacking. The most striking is the lack of any cost-effectiveness study concerning pharmacotherapy, although this is the main intervention in asthma and COPD management. Of all the bronchodilators that are used in current practice, we do not know which ones are the most efficient for patients exhibiting particular clinical symptoms. We do not know whether the extra benefits of combined bronchodilator and corticosteroid therapy compared to bronchodilator 
monotherapy outweigh the extra costs. We argue that - in addition to the relatively large number of effectiveness studies - there is a need for subjecting these relatively 'low cost' therapies to a thorough economic evaluation, since more than $7 \%$ of total drug sales in the Netherlands are related to asthma and COPD management and because it involves so many patients. In the future, cost-effectiveness evidence will increasingly be asked for as a complement to the clinical evidence of the safety and efficacy of new drugs. Such evidence may influence the pricesetting of new drugs and support cost-reducing therapeutic substitution for already existing drugs.

A second important area in which information is totally lacking is that of diagnostic technologies. For cxample, in some countrics an economic evaluation of spirometry would be of value in guiding decisions about whether or not spirometry should be made available too for general practitioners. Similarly, some countries are considering screening for Alpha-1 Antitrypsin deficiency. This decision should be based on a thorough, perhaps theoretical cost-effectiveness analysis, in particular since the number of patients with Alpha-1 Antitrypsin deficiency is relatively small and replacement therapy is relatively expensive. For information on the costs and benefits of such technologies to be useful, it should become available before the technologies are diffused throughout the health care system (Ref. [50]).

Economic appraisals cannot be expected to have an influence on decision-making until the methodological quality of the studies is adequate. We have shown that there is still room for improvement. It is difficult to compare the results of various cost-effectiveness studies, because the analyses are not conducted according to uniform, accepted methodological standards. As these conditions are being met, economic evaluation studies can contribute to improved efficiency in delivering asthma and COPD care.

Acknowledgements - The authors would like to thank R. M. Kaplan, an anonymous referee and our colleagues $G$. Boas and V. H. Ploum for their comments on an earlier draft, Mr J. P. M. A. van Emmerik for his assistance in preparing this manuscript and Glaxo Netherlands for their financial support.

\section{REFERENCES}

1. American Thoracic Society. Standards for the diagnosis and care of patients with chronic obstructive pulmonary disease (COPD and asthma). Am. Rev. Respiratory Dis. 136, 225, 1987

2. Mölken M. P. M. H. van, Doorslaer E. K. A. van and Rutten F. F. H. Chronic non-specific lung disease in figurcs. Report of a pilot-study (in Dutch). Institute for Medical Technology Assessment, Maastricht, The Netherlands, 1989.

3. Lako C. I. Absenteeism in primary school. Tijdschrift voor Sociale Gezondheidszorg 61, 776, 1983. (In Dutch.)

4. Wever, A. M. J. and Wever-Hess J. Trends in the frequency of hospitalizations due to asthma and COPD in the Netherlands 1980-1988. Nederlands Tijdschrift voor Geneeskunde 135, 659, 1991. (In Dutch.)

5. Bergner M., Hudson L. D., Conrad D. A., Patmont C. M., McDonald G. J., Perrin E. B. and Gilson B. S. The cost and efficacy of home care for patients with chronic lung disease. Med. Care 26, 566, 1988.

6. Campbell-Haggerty M., Stockdale-Woolley R. and Nair S. Respi-Care. An innovative home care program for the patient with chronic obstructive pulmonary disease. Chest. 100, 607, 1991.

7. Clark N. M., Feldman C. H., Evans D., Levison M. J., Wasilewski Y. and Mellens M. D. The impact of health education on frequency and cost of health carc use by low income children with asthma. $J$. Allergy clin. Immunol. 78, 108, 1986.

8. Deter H. C. Cost-benefit analysis of psychosomatic therapy in asthma. J. Psychosom. Res. 30, 173, 1986.

9. Dranove D. An empirical study of a hospital-based home care program. Inquiry 22, 59, 1985.

10. Fireman P., Friday G. A., Gira C., Vierhaler W. A. and Michaels L. Teaching self-management skills to asthmatic children and their parents in an ambulatory care setting. Pediatrics 68, 341, 1981.

11. Gay P. C., Patel H. G., Nelson S. B., Gilles B. and Hubmayr R. D. Metered dose inhalers for bronchodilator delivery in intubated mechanically ventilated patients. Chest 99, 66, 1991.

12. Hay J. W. and Robin E. D. Cost-effectiveness of Alpha-1 Antitrypsin replacement therapy in treatment of congenital chronic obstructive pulmonary disease. Am. J. Pub Hlih 81, 427, 1991

13. Jasper A. C., Mohsenifar Z., Kahan S., Golberg H. S. and Koerner S. K., Cost-benefit comparison of aerosol bronchodilator delivery methods in hospitalized patients. Chest 91, 614, 1987.

14. Lewis C. E., Rachelefsky G., Lewis M. A., de la Sota A. and Kaplan, M. A randomized trial of A.C.T. (Asthma Carc Training) for kids. Pediatrics 74, 478, 1984.

15. Lowson K. V., Bishop J. M. and Drummond M. F. Costing new services: long-term domiciliary oxygen therapy. The Lancet 1, 1146, 1981.

16. McKeon J. L., Saunders A. and Murree-Allen D. Domiciliary oxygen: rationalization of supply in the Hunter region from 1982 1986. Med. J. Aust. 146, 73 , 1987

17. Motwani K. J. and Herring G. M., Home care for ventilatory dependent persons: a cost-effective humane public policy. Hlth Soc. Work 13, 20, 1988.

18. Schmidt C. D., Elliott C. G., Carmelli D., Jensen, R. L., Gengiz M., Schmidt J. C., Tolman E. D. and Clemmer T. P., Prolonged mechanical ventilation for respiratory failure: A cost-benefit analysis. Critical Care Med. 11, $407,1983$.

19. Ström K., Boe J., Herala M., Boman G. and Gustavil A. Assessment of two oxygen treatment alternatives in the home. Int. J. Technol. Assess. Hith Care 6, 489, 1990.

20. Toevs C. D., Kaplan R. M. and Atkins C. J. The costs and effects of behavioral programs in Chronic Obstructive Pulnunary Disease. Med. Care 22, 1088, 1984.

21. Wilson-Pessano S. R., Scamagas P., Arsham G. M., Chardon L., Coss S., German D. F. and Hughes G. W. An Evaluation of approaches to asthma self-management education for adults: the AIR/Kaiser-Permanente study. Health Educ. Q. 14, 333, 1987.

22. Windsor R. A., Bailey W. C., Richards J. M., Manzella B., Soong S. J. and Brooks M. Evaluation of the efficacy and cost effectiveness of health education methods to increase medication adherence among adults with asthma. Am. J. publ. Hlth 80, 1519, 1990.

23. Wright R. W., Larsen D. F., Monie R. G. and Aldred R. A. Benefits of a community-hospital pulmonary- 
rehabilitation program. Respiratory Care 28, 1474, 1983.

24. Scheffler R. M. and Delaney M. The implications of cost-effectiveness analysis of medical technology. Background paper \#2: case study of medical technologies. Case study \# 12: assessing selected respiratory modalities: trends and relative costs in the Washington DC area. U.S. Government Printing Office, Washington DC, 1981.

25. Williams S. J. Chronic respiratory illness and disability: a critical review of the psychosocial literature. Soc. Sci. Med. 28, 791, 1989.

26. Drummond M. F., Stoddart G. L. and Torrance G. W. Methods for the Economic Evaluation of Health Carefi Programmes. Oxford University Press, Oxford, 1987.

27. Guyatt G., Drummond M., Feeny D., Tugwell P., Stoddart G., Haynes R. B., Bennett K. and Labelle R. Guidelines for the clinical and economic evaluation of health care. Soc. Sci. Med. 22, 393, 1986.

28. Drummond M. F. (Ed.) Economic Appraisal of Health Technology in the European Community. Commission of the European Communities. Health Services Research Series No. 2. Oxford University Press, Oxford, 1987.

29. Hodgkin J. E. Pulmonary rehabilitation. Clinics Chest Med. 11, 447, 1990.

30. Petty T. L. Home oxygen therapy. Mayo Clin. Proc. 62 , $841,1987$.

31. Postma D. S., de Vries K., Koëter G. H. and Sluiter H $J$. Independent influence of reversibility of airflow obstruction and non-specific hyperreactivity on the longterm course of lung function in chronic airflow obstuction. Am. Rev. Respir. Dis. 134, 276, 1986

32. Atkins C. J., Kaplan M., Timms R. M., Reinsch S. and Loftback K. Behavioral exercise programs in the management of chronic obstructive pulmonary disease J. Consult. clin. Psychol. 52, 591, 1984.

33. Williams S. J. and Bury M. R. Impairment, disability and handicap in chronic respiratory illness. Soc. Sci. Med. 29, 609, 1989.

34. Jones $\mathbf{P}$. W. Quality of life measurement in chronic lung disease. J. Drug Ther. Res. 13, 181, 1988.

35. McSweeny A. J., Heaton R. K., Grant I., Cugell D., Solliday N. and Timms R. Chronic obstructive pulmonary disease; socio-emotional adjustment and life quality. Chest 77, 309, 1980.

36. Guyatt G. H., Berman L. B., Townsend M., Pugsley S and Chambers L. W. A measure of quality of life for clinical trials in chronic lung disease. Thorax 42, 773, 1987.
37. Kaptein A. A., Dekker F. W., Dekhuijzen P. N. R., Wagenaar J. P. M. and Janssen P. J. The value of the 12-minutes walking test in patients with chronic obstructive pulmonary disease, II walking in the laboratory and walking in daily life. Ned Tijdschr Geneeskd 131, 1717, 1987. (In Dutch.)

38. Schrier A. C., Dekker F., Kaptein A. A. and Dijkman J. H. Quality of life in elderly patients with chronic nonspecific lung disease seen in family practice. Chest 89, 894, 1990 .

39. Schayck C. P. van, Rutten M. P. M. H., Doorslaer E. K. A. van, Folgering H. and Weel C. van Two-year bronchodilator treatment in patients with mild airflow obstruction: contradictory effects on lung function and quality of life. Chest. In press.

40. Guyatt G. H., Townsend M., Berman L. B. and Pugsley S. O. Quality of life in patients with chronic airflow limitation. Br. J. Dis. Chest 81, 45, 1987.

41. Kaplan R. M., Atkins C. J. and Timms R. Validity of a quality of well-being scale as an outcome measure in chronic obstructive pulmonary disease. J. Chronic Dis. 37, 85, 1984.

42. Kaplan R. M., Bush J. W. and Berry C. C. Health status: types of validity and the index of well-being. Hlth Services Res. 11, 478, 1976.

43. Donaldson C., Atkinson A., Bond J. and Wright $\mathbf{K}$. Should Qalys be programme-specific? J. Hlth Econ. 7, $239,1988$.

44. Drummond M. F., Ludbrook A., Lowson K. and Stcelc A. Studies in Economic Appraisal in Health Care., Vol. 2 University Press, Oxford, 1986.

45. Martens L. L. M. and Doorslaer E. K. A. van Dealing with discounting. Int. J. Techn. Assess. Hith Care 6, 139, 1990.

46. Johanneson M. and Levin L. A. Comments on "Dealing with discounting". (Letter to the editor). Int. J. Techn. Assess. Hlth Care 7, 649, 1991.

47. Doorslaer E. K. A. van and Martens L. L. M. On the discounting of gains of life expectancy. Reply to Johanneson and Levin. Int. J. Techn. Assess. Hlth Care 7, 653, 1991

48. Drummond M. F. Principles of Economic Appraisal in Health Care. Oxford University Press, Oxford, 1980.

49. Drummond M. F. and Stoddart G. L. Economic analysis and clinical trials. Controlled clin. Trials 5, 115, 1984.

50. Rutten F. F. H., Greep J. M. and Haan G. H. M. G Public control of the diffusion of health technology. In The Economics of Medical Technology, Proceedings of an Int. Conf. on Economics of Medical Technology (Edited by Rutten F. F. H. et al.). Springer, Berlin, 1988. 